\title{
Norois
}

Environnement, aménagement, société

$223 \mid 2012$

Villes petites et moyennes

\section{Se reconnaître et se revendiquer comme « ville moyenne » : postures d'acteurs locaux en France, en Espagne et au Portugal dans le cadre du programme INTERREG « ATI »}

Recognizing and asserting its city as a medium size town: a local actor's choice in France, Spain and Portugal in european programme INTERREG «ATI »

\section{Frédéric Tesson}

\section{(2) OpenEdition}

Journals

Édition électronique

URL : https://journals.openedition.org/norois/4193

DOI : 10.4000/norois.4193

ISBN : 978-2-7535-2043-1

ISSN : 1760-8546

Éditeur

Presses universitaires de Rennes

Édition imprimée

Date de publication : 30 mars 2012

Pagination : $31-42$

ISBN : 978-2-7535-2041-7

ISSN : 0029-182X

Référence électronique

Frédéric Tesson, « Se reconnaître et se revendiquer comme « ville moyenne » : postures d'acteurs

locaux en France, en Espagne et au Portugal dans le cadre du programme INTERREG «ATI »», Norois [En ligne], 223 | 2012, mis en ligne le 28 février 2014, consulté le 13 janvier 2022. URL : http:// journals.openedition.org/norois/4193 ; DOI : https://doi.org/10.4000/norois.4193 

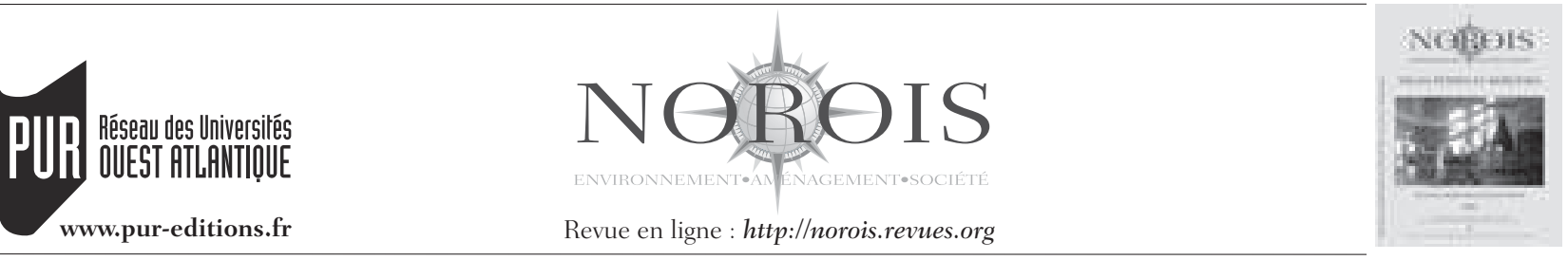

\title{
Se reconnaître et se revendiquer comme «ville moyenne » : postures d'acteurs locaux en France, en Espagne et au Portugal dans le cadre du programme INTERREG «ATI »
}

\author{
Recognizing and Asserting its City as a Medium Size Town: A Local Actor's Choice in France, \\ Spain and Portugal in European Programme INTERREG "ATI"
}

\author{
Frédéric Tesson
}

SET-IRSAM-UMR 5603 CNRS, Université de Pau et des Pays de l'Adour, Domaine universitaire - 64000 PAU, France,(frederic.tesson@univ-pau.fr)

Résumé : La ville moyenne est un objet difficile à identifier pour les chercheurs. Ceux réunis dans le cadre du programme ORATE 1.4.1 le démontrent parfaitement et d'autres avant eux avaient fait état de cette difficulté à établir une catégorie opératoire. Mais au-delà de cette quête, qui concerne un public de chercheurs soucieux de construire une catégorie pour comprendre une réalité difficilement accessible, qu'en est-il de la posture des acteurs de ces villes? Nous la questionnons ici à travers l'expérience d'un programme INTERREG intitulé « attractivité des territoires et innovation : le cas des villes moyennes ». Mené entre 2005 et 2007 , il était piloté par la CCI Pau-Béarn et regroupait onze partenaires français, espagnols et portugais. Au-delà des travaux propres à chaque partenaire, il a permis de faire émerger des questions susceptibles d'alimenter le débat, d'une part autour de la définition de la ville moyenne, quand bien même il s'agit ici de regards issus des représentations de ces acteurs, d'autre part sur les atouts que peut représenter la revendication de l'appartenance à cette catégorie pour certains acteurs locaux. Ainsi, il s'agira de montrer que la définition de la ville moyenne proposée localement par les acteurs est contingente de la position de l'acteur qui s'exprime (depuis la métropole ou depuis la ville moyenne elle-même), mais aussi d'autre part que la revendication « ville moyenne » dans le discours de ces mêmes acteurs correspond à la reconnaissance d'un pouvoir de distinction que ce statut peut leur conférer. Nous souhaitons montrer que détourner le regard pour écouter les acteurs sur ce qu'est une ville moyenne permet de révéler des leviers fondamentaux de sa définition.

\begin{abstract}
Researchers have difficulty to identify the medium towns. The ESPON 1.4.1 reaserch program about "smal and medium towns" shows perfectly this problem, and other researchers before them recognized this difficulty in establishing an operational category. But beyond the attempt to build a reasearch category, what about the position of actors in these cities? We approached this question through the experience of INTERREG european program entitled «attractiveness of regions and innovation: the case of medium towns.» Conducted between 2005 and 2007, it was led by Pau-Béarn CCI with eleven europeans partners (French, Spanish and Portuguese). This program has helped bring out issues that may contribute to the debate. First of all it concerns the definition of the medium town from the representation of actors who live it. Second, they suggest that the benefits may be the claim of belonging to this category for some local actors. Thus, it will be first to show that the definition of the medium town proposed by local people is contingent on the position of the actor who speaks (from the county seat or from the medium town itself), secondly that the claim «medium town" in the speech of these actors is the recognition of a power of distinction that this status can give them. We want to show that look the actors to listen to what a medium town can reveal fundamental levers of its definition.
\end{abstract}


Mots clé : ville moyenne - INTERREG SUDOE - hiérarchie - domination - acteurs locaux

Keywords: medium town - INTERREG SUDOE - hierarchy domination - local actor

\section{INTRODUCTION}

\section{De la vanité du débat scientifique}

La ville moyenne est un objet qui se définit par défaut, dont les caractéristiques dépendent de ce qu'elle n'est pas. Ainsi, le débat sur la ville moyenne consisterait essentiellement à définir ce qu'est une " grande ville » et ce qu'est une " petite ville», la ville moyenne s'installant dans le creux dessiné par ces deux pôles. Mais la limite inférieure est souvent éliminée dans le débat si on en croit la réflexion menée dans le cadre du programme européen ORATE ${ }^{1}$, dont le lot 1.4.1 (ORATE/ESPON, 2006) portait sur le rôle des petites et moyennes villes (SMESTO). Et on a beau nous expliquer que l'indicateur démographique n'est pas essentiel, que les choses se jouent ailleurs, dans le niveau de services, dans la dépendance aux centres, etc., c'est bien en termes démographiques que les chercheurs finissent toujours par délimiter leur périmètre d'investigation (Santamaria, 2000; DIACT, 2007).

Ce débat nous intéresse peu au final tant il apparaît vain. Car en tout état de causes, le caractère moyen d'une ville est toujours relatif. À l'échelle planétaire, nos métropoles françaises paraissent bien moyennes, tant en termes démographiques que du point de vue de leur niveau de fonctions et de la domination dont elles font l'objet. Parler de ville moyenne revient à choisir de circonscrire le regard à l'échelle européenne au maximum, mais plus surement à l'échelle régionale. Ne peut-on pas dire qu'une ville est moyenne relativement à la domination qu'une ville plus importante, que l'on pourra qualifier de métropole, exerce sur elle, et à la domination qu'elle est capable d'exercer sur des villes plus petites? Parler de ville moyenne nous projetterait dans un schéma « christallérien » (Christaller, 2005 [1933]) qui seul permet d'accorder du crédit à une définition plus fonctionnelle que démographique de ce type urbain. Ainsi, nous préférons parler de ville intermédiaire, cela permet d'évacuer la

1. Pour télécharger le rapport, le résumé et l'évaluation : [http://www.umsriate.fr/141.php]. prégnance de l'indicateur démographique puisque ce qui la caractérise devient le rapport de domination qu'elle subit d'un côté et celui qu'elle exerce de l'autre.

\section{Déplacer le débat dans le champ de l'action : la détermination et l'autodé- termination de la ville moyenne ne nous donnent-elles pas les clés pour com- prendre ce qu'elle est?}

S'il existe un débat scientifique sur la ville moyenne, un autre est ouvert depuis près d'un demi-siècle dans l'action publique. S'il témoigne des mêmes errances en termes de définition, il semble plus intéressant du fait de la mise évidence d'une réalité vécue plus féconde que les joutes scientifiques. Cette approche qui vise à prendre au sérieux les discours des acteurs n'est pas nouvelle, l'émergence récente du concept de gouvernance se nourrit de ces discours portés par les acteurs sur euxmêmes. Dans cette perspective les politologues ont déjà largement ouvert la brèche avec, notamment, Alain Faure dont les travaux témoignent de cette attention portée à la parole de l'élu (Faure, 2009), que l'on peut élargir à la parole au sujet. C'est ce parti-pris que nous avons choisi ici.

À travers notre propos, nous souhaitons déplacer le débat dans l'action pour tenter de comprendre la manière dont les acteurs locaux mobilisent la catégorie « ville moyenne » pour s'inscrire dans le territoire. En France, les « contrats de villes moyennes », en 1973, ou la politique actuelle des « villes moyennes témoins » lancée par la DATAR, ont entrainé des villes à s'autodéterminer ville moyenne, mais l'Etat avait fixé un cadre démographique qui finalement formatait l'engagement, imposait à chacun de rentrer dans une fenêtre pour saisir l'opportunité. D'un autre côté, la politique des réseaux de villes, soutenue par la DATAR également, s'affichait comme une réaction à la politique des pôles de croissance prônée par le rapport Guichard (1986). Au final, nous avions pu montrer (Tesson, 1996, 2000) que les villes qui s'en étaient saisies étaient toutes des villes intermédiaires et que les acteurs engagés considéraient que le réseau allait leur permettre de franchir un seuil dans cette intermédiarité. Mais dans ce cas, les villes elles-mêmes ne s'autoproclamaient 
pas villes moyennes ou villes intermédiaires, c'est l'analyse du chercheur qui révélait cette constante.

C'est pourquoi nous avons souhaité appuyer notre propos sur un programme INTERREG IIIb SUDOE, piloté par la CCI Pau-Béarn, dont le titre : «attractivité des territoires et innovation : le cas des villes moyennes » conditionnait l'engagement des partenaires à l'appartenance auto-décrétée à cette catégorie. Que nous dit ce type d'engagement sur la ville moyenne? N'est-il pas possible de dégager, à travers cette autodétermination, quelques leviers d'une définition opératoire qui permettraient d'orienter les hypothèses des chercheurs? Deux types de regards apparaissent dans ce programme. Le premier s'inscrit dans une logique centralisée, depuis la métropole, et pose sur la ville moyenne un regard en termes de domination. Le second concerne les partenaires qui ont répondu en leur nom, en se revendiquant ville moyenne, alors même que leur ville n'appartient pas à la catégorie de manière évidente. Nous montrerons que ces deux attitudes révèlent des éléments intéressant apportant des arguments forts pour une approche renouvelée de la ville moyenne. Mais avant d'analyser ces deux dimensions, nous présenterons dans un premier temps le programme INTERREG dont il est question pour en comprendre les objectifs et en connaître les partenaires.

\section{Programme INTERREG « ATTRACTIVITÉ DES TERRITOIRES ET INNOVATION : LE CAS DES VILLES MOYENNES » : LA DIVERSITÉ DES OBJECTIFS ET DES PARTENARIATS}

En 2004, la CCI Pau Béarn a proposé un projet de programme INTERREG dans le cadre du volet IIIb SUDOE, sur l'attractivité des territoires et l'innovation dans les villes moyennes. Cet appel à proposition a débouché sur neuf candidatures de structures en Espagne et au Portugal. Nous avons été intégrés dans le programme en tant que partenaire dans le cadre d'une association entre le SET (UMR 5603, CNRS/Université de Pau et des Pays de l'Adour) et ADES (UMR 5185, CNRS/Université de Bordeaux 3). Ainsi, nous avons pu suivre l'ensemble de la démarche et des actions menées par les différents partenaires. Pour ce qui nous intéresse ici, nous souhaitons revenir sur un des objectifs qui a été le nôtre au début du programme, à savoir : tenter de créer une culture commune du programme et ainsi comprendre quels étaient les attentes de chacun. Nous avons donc commencé par définir les principaux termes du programme, dont celui de ville moyenne.

\section{Les objectifs généraux}

Le contexte proposé par la CCI Pau-Béarn est clairement celui du défaut de compétitivité des villes moyennes face à l'injonction d'innovation faite aux territoires par les directives européennes. Il est donc proposé dans ce programme de repérer, évaluer et partager les approches innovantes des villes moyennes pour faire émerger des spécificités et les ériger en bonnes pratiques.

«Le projet Attractivité du Territoire et Innovation part de l'hypothèse qu'un territoire est d'autant plus attractif qu'il accueille les entreprises innovantes. L'innovation étant effectivement une condition indispensable pour faire face à la concurrence et aux nouveaux marchés.

En parallèle, les villes moyennes "oubliées" des grandes directions de l'aménagement du Territoire peuvent, si elles s'organisent en fonction de cet enjeu, développer les conditions de leur identité. Situées dans des territoires intermédiaires, et à condition de s'organiser en réseau avec des villes similaires en rapport avec l'objectif de compétitivité, elles peuvent, grâce à ce maillage, rivaliser ou du moins être reconnues par les grandes métropoles, en créant des projets pilotes de plateformes technologiques; elles couvriront ainsi les besoins des entreprises qui pourraient avoir la tentation de se délocaliser, et du tissu des PME existantes en majorité dans ces villes et ces territoires intermédiaires qui pourront ainsi rivaliser dans le marché de la compétitivité. » (Extrait du formulaire de candidature du projet ATI)

L'objectif poursuivi concerne la connaissance des villes et leur reconnaissance. Il s'est agi, dans le cadre de ce programme, de comprendre l'organisation des villes moyennes, leur potentiel d'innovation et leur capacité d'attractivité. Il apparaît également, et nous en verrons l'importance par la suite, que l'affirmation de la prégnance de l'identité de la ville moyenne par le chef de file constitue une entrée particulièrement sensible chez les partenaires engagés. 
Un programme INTERREG de cet ordre combine deux dimensions. La première concerne les actions que le partenaire va mettre en œuvre en son nom propre dans le cadre du programme, nous reviendrons plus loin sur cette question. La seconde consiste à promouvoir le programme dans sa dimension transnationale en poursuivant un objectif commun susceptible d'apporter une plus-value à l'ensemble des partenaires. Le chef de file est garant de la réalisation de cet objectif et, dans le cas présent, nous avons été chargés d'animer cette dimension transnationale. Il s'agissait de créer une culture du programme pour dégager de grandes orientations susceptibles de donner lieu à des mutualisations. Ces objectifs généraux étaient rédigés en ces termes :

«-Améliorer la connaissance des territoires afin de concrétiser les paramètres de leur attractivité,

- Rechercher les passerelles entre recherche universitaire et recherche appliquée à l'industrie dans le réseau des villes moyennes du Sud Ouest européen

- Favoriser des axes de développement économique et les potentiels locaux,

- Permettre aux villes moyennes d'entrer dans un réseau d'innovation afin de polariser leurs ressources et leur potentiel de développement,

- Permettre l'émergence de projets pilotes inscrits dans les programmes de développement des villes moyennes,

- Créer des réseaux nécessaires pour diffuser la culture de l'innovation dans des espaces non métropolitains et rendre ces territoires attractifs. » (Extrait du formulaire de candidature du projet ATI)

Ainsi, chaque partenaire devait travailler pour le collectif en réalisant un diagnostic fin du territoire sur lequel il développait ses activités afin de connaître les modalités de relations recherche/ entreprise, les structures de gouvernance de ces liens, les potentiels et les projets existants... Ce travail avait vocation à permettre une mutualisation des pratiques et une meilleure efficacité des réseaux.

\section{Les partenaires}

L'intérêt de ce programme dans le cadre des questions que nous souhaitons poser ici, réside dans la multiplicité et dans la diversité des partenariats. Lorsqu'en 2004 la CCI Pau-Béarn lance son appel à participation sur le site internet du programme INTERREG SUDOE, il n'est pas surprenant que l'essentiel des candidats se reconnaissant dans la démarche soit des structures du même ordre en Espagne et au Portugal. Ainsi on retrouve plusieurs chambres de commerce ou équivalents (Communauté Valencienne et Aragon en Espagne; CEC et AERSET au Portugal) parmi les partenaires définitifs du programme tel qu'il a été accepté. Il est intéressant de noter que certains de ces partenaires sont entrés dans le programme accompagnés de structures d'ingénierie, que celles-ci soit des structures privées (l'institut technologique d'Aragon), parapubliques (IERU à Coimbra) ou publiques (les laboratoires ADES et SET en Aquitaine). À ces tandems entreprise/recherche il faut ajouter les candidatures isolées de l'université de Gérone en Espagne et surtout deux municipalités espagnoles : Dos Hermanas et Torrelavega, dont l'engagement est porté par la sphère politique.

Cette structure partenariale promettait une forte capacité d'expertises locales et un bon potentiel d'échanges. Si Torrelavega, Dos Hermanas et Gerone avaient une approche restreinte à leur propre entité, les autres structures permettaient des approches plus territoriales : Aragon, communauté valencienne (province d'Alicante), région centre Portugal et Aquitaine (Pyrénées Atlantiques) (figure 1).

\section{La diversité des objectifs spécifiques}

Le potentiel d'investigation réuni par le programme allait être nuancé par les objectifs individuels de chaque partenaire. En effet, si une convergence apparaissait entre les partenaires français, aragonais et portugais, sur la compréhension globale de l'organisation de ces villes, les autres partenaires poursuivaient des objectifs plus restreints. Ainsi, Dos Hermanas était entrée dans le programme essentiellement pour mettre en œuvre un congrès d'entrepreneurs locaux intitulé "l'innovation ne peut pas attendre » qui en était à sa seconde édition. Torrelavega et Gerone avaient comme objectif le financement d'infrastructures innovantes de type « incubateur » ou «pépinières d'entreprises » ou l'équipement informatique de salles de cours. Quant à la chambre de commerce de la communauté valencienne, son approche était celle des clusters et 


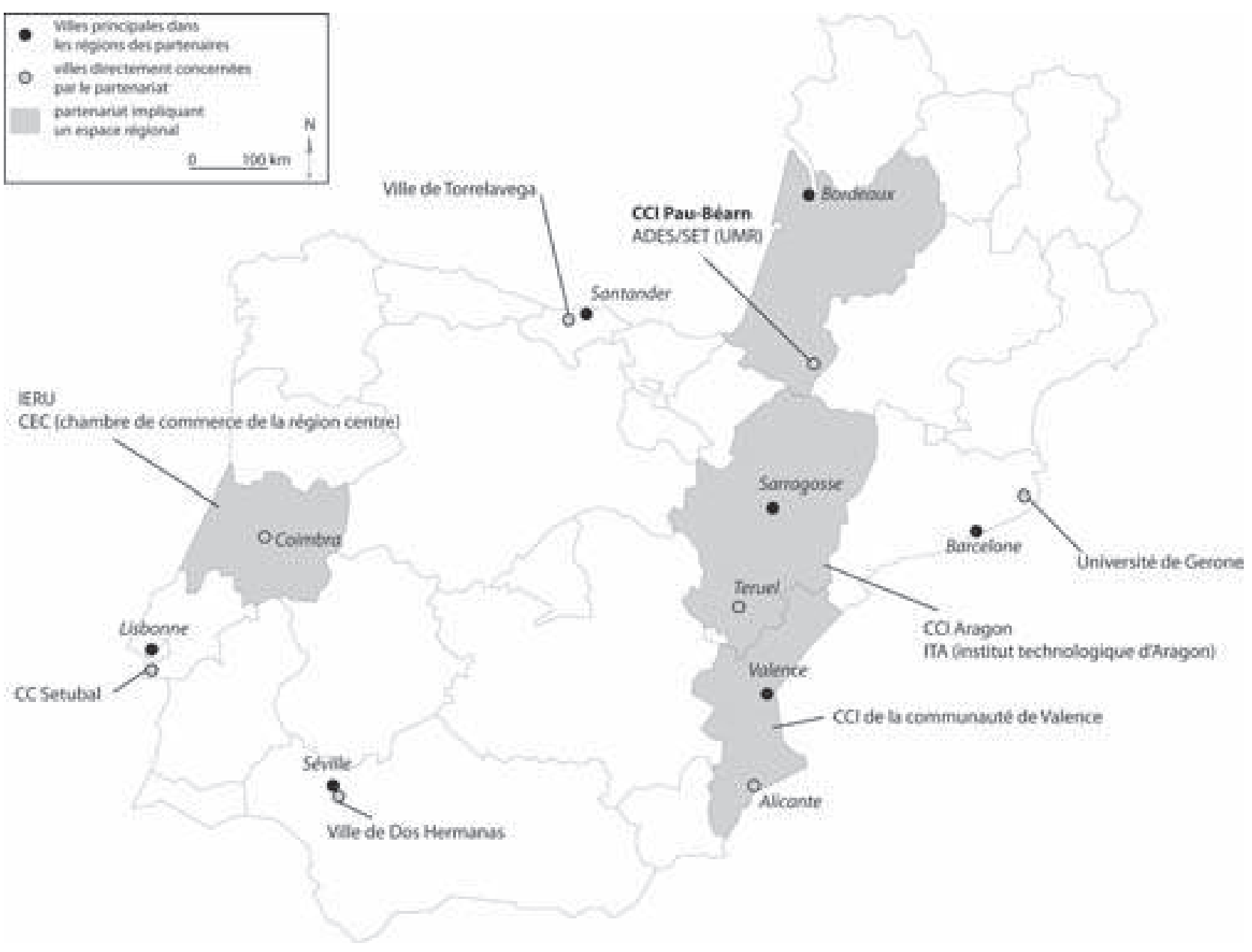

Figure 1 : Carte de localisation des partenaires du programme INTERREG IIIb SUDOE : «Attractivité des Territoires et Innovation dans les Villes Moyennes»

Cette carte montre la localisation des partenaires et l'espace pris en considération dans le partenariat.

Localization map of programme members (INTERREG SUDOE "attractiveness of regions and innovation : the case of medium towns") This map shows the localization of progamme members and the scale of places selected in the partnership.

comptait sur ce programme pour animer sa politique locale de cluster autour d'Alicante.

Il apparaît dès lors que, dans la mesure où les projets locaux étaient aussi disparates, il était peu probable de voir émerger une réelle coopération transnationale. Le propos n'est pas ici de faire le procès d'un programme INTERREG, mais simplement de montrer que les projets eux-mêmes sont évocateurs de la compréhension globale de ce qu'est une ville moyenne.

\section{LA VILLE MOYENNE VUE DEPUIS LE CENTRE : LA MISE EN PERSPECTIVE D'UNE DÉFINITION DE LA VILLE MOYENNE PAR LA HIÉRARCHIE}

Lorsque l'on parle de Ville moyenne à Valence ou à Saragosse, il est évident que les images projetées sont différentes. Les contextes régionaux sont très éloignés et la taille même des villes ne semble pas pouvoir permettre une quelconque comparaison. Ce qui les rassemble ici, c'est le fait qu'en Aragon et dans la communauté valencienne, la ville moyenne est observée depuis la ville centre par des institutions de type chambres de commerces dont l'aire 
d'influence est régionale. Il s'agit pour elles de s'emparer de la question qui pose, pour chaque cas, un problème majeur mais distinct, en le traitant à partir du centre. Ce regard descendant sur le cadre régional renvoie à l'idée d'aménagement du territoire, quand bien même le regard est porté par des chambres consulaires. Ainsi, depuis Sarragosse ou depuis Valence, la ville moyenne est considérée au sein de la hiérarchie. Ce type de ville représente la ville dominée à laquelle il est indispensable, dans un souci d'équité, de donner les moyens de se développer. Si la problématique n'est pas du tout la même, si les actions menées sont bien différentes et si au final ils n'ont guère appris les uns des autres, les discours des partenaires nous permettent de donner du crédit à une approche de la ville moyenne en termes de rapports de domination. La ville moyenne serait celle qui est dominée d'un côté mais qui domine de l'autre, quel que soit son poids démographique.

\section{Teruel vs Alicante : l'intermédiarité comme seul point commun}

En Aragon, nous nous appuierons sur l'exemple de Teruel qui a été une des villes particulièrement mobilisée dans le travail mené par la chambre de commerce et par l'ITA. Pour l'essentiel, la problématique a d'ailleurs porté sur le Sud de l'Aragon, marqué par une impression de désert vertigineuse si on se place du point de vue de la capitale. Côté valencien, c'est Alicante qui a été le terrain d'application privilégié du travail du partenaire. Or, indépendamment du travail mené, il est difficile d'imaginer que les problématiques de Teruel puissent être les mêmes que celles d'Alicante.

En effet, si les deux villes sont capitales de province, la province de Teruel, située dans une zone aride au Sud de l'Aragon, compte moins de 150000 habitants $^{2}$. La ville de Teruel en abrite un peu plus de 35000 et Alcaniz, la seconde ville, 16000 . Après on ne trouve dans la hiérarchie qu'une ville de plus de 5000 âmes et sept de plus de 2000 . Le rapport de population entre Teruel et Sarragosse est de 1 à 20 alors que Teruel est pourtant la troisième ville d'Aragon (Huesca comptant 55000 habitants). La liaison entre Teruel et Sarragosse, si elle s'est beaucoup améliorée, demande tout de même

2. Les chiffres de population sont ceux de 2009 (source INE, Instituto nacional de estatistica, équivalent espagnol de l'INSEE).
2 heures d'autoroute pour parcourir les $186 \mathrm{~km}$ qui les séparent. Les relations ferroviaires sont, quant à elles, très limitées (quatre aller/retour par jour en semaine, trois le dimanche) et tout aussi lentes (deux heures et six minutes pour le voyage le plus rapide et deux heures et quarante minutes pour le plus lent).

La province d'Alicante se situe clairement à un autre niveau puisqu'elle était forte de près de 2 millions d'habitants en 2009. La ville d'Alicante en compte plus de 330000 (plus du double de l'ensemble de la province de Teruel). Elle fait partie d'une des 15 conurbations espagnoles recensées par l'INE associée à Elche (230 000 hbts) et forme un ensemble urbain de près de 800000 habitants. La province compte 36 municipalités de plus de 10000 habitants. Le rapport de population entre Alicante et Valence est de 1 à 2,5 en ce qui concerne les municipalités et de 1 à 2 pour les conurbations urbaines puisque celle de Valence affiche plus d'1,7 millions d'habitants. Les deux villes sont également séparées par 2 heures d'autoroute mais le voyage en train est un peu plus rapide (entre $1 \mathrm{~h} 30$ et $2 \mathrm{~h} 15$ ), pour une dizaine d'aller-retour par jour avec des horaires calibrés pour des navetteurs potentiels le matin et le soir.

Il apparaît, avec ces quelques chiffres, qu'imaginer que l'un puisse apprendre de l'autre sur la problématique de la ville moyenne peut sembler illusoire. En revanche, ces deux exemples corroborent l'idée que la ville moyenne pourrait être définie par la distance au centre d'une part, les deux villes sont à 2 heures de leur capitale et, d'autre part, par leur place dans la hiérarchie puisqu'elles occupent finalement le même rang. Ainsi, ce regard depuis le centre montre que réfléchir sur la ville moyenne depuis la métropole, consiste à se préoccuper de l'équilibre des villes de niveau immédiatement inférieur, quelle que soit leur taille. Pour autant, les actions menées par les deux partenaires ont été très différentes et il est difficile de définir ici une préoccupation commune.

\section{Le développement des petits noyaux urbains versus la politique des clusters}

Du côté aragonais, la problématique majeure concernait l'analyse des processus de développement possibles et surtout la compréhension du rôle que jouent ces villes, et principalement Teruel, dans 
l'organisation régionale. Comment insuffler une dynamique, pour les entreprises mais aussi plus globalement pour le territoire? La question de l'innovation relevait donc plus des questions de gouvernance et d'une innovation ordinaire pour reprendre l'expression de Norbert Alter (2005 [2000]). Ainsi, l'action principale du programme a consisté à organiser à Teruel un colloque intitulé « les noyaux urbains petits et moyens : technologie et innovation comme facteurs de développement ${ }^{3}$ ».

Ainsi, le programme ATI a été l'occasion pour la chambre de commerce d'Aragon de confronter les regards sur une catégorie très spécifique de ville moyenne et de mettre Teruel dans la lumière. Il faut souligner la volonté de l'ITA d'enrichir sa capacité d'analyse en s'ouvrant à des regards extérieurs et universitaires (français et portugais). On a pu sentir une forme d'urgence pour le développement de ce type urbain, quand bien même cette urgence était décrétée par le centre et ne semblait pas faire réellement écho à une préoccupation locale. Pour les partenaires aragonais, positionner Teruel (mais aussi Caltayud ou Alcaniz) comme ville moyenne n'avait rien d'évident lors des premiers contacts, et leur volonté était clairement de faire admettre aux partenaires l'importance de les considérer ainsi. Eux aussi, quelque part, cherchaient une légitimité à ces villes pour les faire entrer dans une catégorie dont l'image est considérée comme positive. Il faut ajouter que ne pas considérer Teruel ou Calatayud comme des villes moyennes reviendrait à dire que l'Aragon ne compte pas de villes moyennes, ce qui n'est pas pensable d'un point de vue des acteurs locaux, économiques dans le cadre du programme ATI, mais aussi politiques comme nous avons pu le remarquer lors du colloque de Teruel.

En ce qui concerne Alicante, la problématique était tout autre. Centrée sur l'innovation technologique dans les entreprises (PME et PMI), la chambre de commerce tenait à utiliser le programme pour mettre en scène et valoriser la politique de clusters menée à Alicante mais aussi dans toute la province. Il s'agissait donc de promouvoir par le biais d'INTERREG, la dimension organisationnelle de sa politique en montrant comment ils étaient parvenus à mettre en réseau des entreprises, dans des configurations finalement assez peu inno-

3. « Los nucleos urbanos pequeños y medios : tecnologia e innovacion como factores de desarollo », Teruel les 26 et 27 octobre 2006 vantes puisqu'héritées des modèles désormais classiques de l'Emilie Romagne ou du choletais racontés de multiples fois, déjà repris, notamment à l'époque des réseau de villes (DATAR, 1991), et qui nourrissent aujourd'hui la politique française des pôles de compétence dans une version polarisée. Cluster du jouet, du marbre, ou de la chaussure, les études et publications de la chambre de commerce de Valence ont montré que le programme ATI a essentiellement été utilisé comme une vitrine européenne pour afficher leur technicité et montrer Alicante comme ville moyenne attractive et dynamique. Il faut ajouter que le travail fait sur Alicante a également permis de présenter le rapport de domination que cette ville, considérée ici comme moyenne, exerce sur une nuée de villes, considérées comme petites, au sein desquelles se développent les entreprises structurant les clusters. Or des villes comme Ibi, dans le cluster du jouet, compte près de 25000 habitants ou Monovar, dans celui du marbre, plus de 13000 , et sont situées à $40 \mathrm{~km}$ d'Alicante. L'idée de s'affranchir de la taille pour se focaliser sur la place dans la hiérarchie semble prendre corps ici.

\section{Pour conclure : domination et distance au centre}

Vue du centre et à l'échelle régionale, la ville moyenne est celle qui est dominée par la métropole et qui domine son hinterland mais aussi les petites villes. La question démographique disparaît totalement puisqu'entre Teruel et Alicante le point commun réside uniquement dans la place que chacune de ces villes occupe dans la hiérarchie régionale. Cette analyse nous montre aussi l'importance de la distance à la métropole dominante qui reprend le schéma christallerien. Cette distance apparaît comme une condition d'émergence et une condition de maintien dans le statut de ville moyenne. Seule une ville à une certaine distance de la métropole semble pouvoir être qualifiée de ville moyenne. Ainsi, on peut voir Elche et ses 200000 habitants englobés dans la conurbation d'Alicante pourtant considérée ici comme ville moyenne relativement à Valence. 


\section{« JE SUIS UNE VILLE MOYENNE DONC JE SUIS!" : DOS HerMaNAS et TorrelaVEGa, DEUX VILLES SATELLITES EN QUÊTE D'IDENTITÉ}

Un élément fondamental qui ressort de l'analyse de ce qu'est une ville moyenne pour les partenaires concerne l'identité que ces villes recèlent. Cette identité est mise en avant par les institutions économiques régionales comme un atout pour des villes comme Teruel, Calatayud, Alicante, Coimbra, etc. L'idée que la ville moyenne possède une identité propre construite dans l'histoire, induisant une reconnaissance depuis l'extérieur et dont les habitants se sentent investis, est bien ancrée dans les esprits des partenaires. Ce principe est d'autant plus fort que certains partenaires se sont impliqués en tant que ville, le partenariat étant porté par les élus. Ces derniers soulignent le manque d'identité de leur cité. Il apparaît assez clairement que les deux villes qui se sont engagées en tant que telles dans le programme, ont rejoint le projet pour ce qu'il leur apportait de reconnaissance. Torrelavega et Dos Hermanas, à la manière des villes de banlieue ou des villes satellites, n'existent que relativement à la ville centre qui les domine. Ainsi leurs problématiques en termes d'attractivité et d'innovation sont totalement différentes de celles vues précédemment puisqu'elles bénéficient de l'effet d'entrainement de la ville métropole (Santander pour la première et surtout Séville pour la seconde). Totalement intégrées dans leur orbite, elles ne rencontrent aucun problème d'attractivité puisqu'elles sont justement sur le front de la délocalisation périphérique des activités économiques et de la fonction résidentielle. Elles bénéficient même parfois d'équipements très spécifiques qui leur donnent une lisibilité au sein de l'ensemble urbain sans pour autant parvenir à les installer en tant que ville sur la carte européenne. Qui connaît Dos Hermanas ou Torrelavega au-delà de leur région? On identifie Teruel et ses 30000 âmes et peu Dos Hermanas et ses 120000 habitants. On retrouve là, en creux, une des clés fondamentales de la définition de la ville moyenne mise en lumière dans le point précédent : le nécessaire isolement relatif, l'existence pour et par elle-même qui renvoie en fait à un schéma hiérarchique.

L'hypothèse que nous formulons ici, replace la question au niveau de l'autodétermination d'une ville en tant que ville moyenne. Pour les édiles de ces deux municipalités, l'intégration à ce programme INTERREG a permis d'affirmer une existence remise en cause par la proximité de la métropole.

\section{Dos Hermanas : la revendication «ville moyenne " comme possibilité d'existence}

Le cas de Dos Hermanas est symptomatique de celui des communes démographiquement développées du fait de leur intégration dans une aire urbaine importante. Il pourrait être rapproché, à la morphologie près, du cas de Mérignac qui s'affiche fièrement comme troisième ville d'Aquitaine avec ses 67000 habitants, revendiquant un aéroport international et des activités de haute technologie sur son territoire.

Dos Hermanas, avec 122000 habitants en 2009 est la seconde municipalité de la province de Séville, assez loin tout de même des 703000 habitants de la capitale. Les deux municipalités sont limitrophes (figure 2), au point qu'un contesté territorial historique les opposent, Séville ayant annexé, au début de la période franquiste, une partie du territoire de Dos Hermanas. La relative discontinuité morphologique, en voie d'être comblée par de vastes projets immobiliers, ne trouble pas l'impression de proximité entre les deux entités (7 à $8 \mathrm{~km}$ ). La ville est bien entendu intégrée dans l'aire métropolitaine de Séville, organisée en comarca, qui compte près d'1,5 millions d'habitants.

Dos Hermanas profite largement de sa proximité avec Séville puisque, dans un contexte général de croissance démographique pour l'Andalousie, la capitale n'a gagné que 3000 habitants depuis l'an 2000 alors que Dos Hermanas a progressé de 30000 habitants dans la même période. Dotée d'une vaste zone industrielle, la municipalité a profité de ce programme INTERREG pour mettre en avant la dimension innovante de son industrie. Reprenant les poncifs du I+D $+\mathrm{I}^{4}$, nés de la stratégie de Lisbonne qui impose l'innovation comme norme (avec tout le paradoxe de l'expression), on ne peut pas affirmer que les entreprises Nazarenas sont plus innovantes que les autres. Cela dit, la dynamique impulsée par la puissance publique, associée aux aides européennes largement utilisées, permettent

4. Investigacion, desarrollo e innovacion. 
Figure 2: Situation de Dos Hermanas relativement à Séville

La municipalité de Dos Hermanas revendique son statut de ville moyenne pour exister dans son rapport à Séville alors même que son développement est totalement dépendant de la capitale andalouse.

Localization of the municipality of Dos Hermanas in comparison with Seville location

Dos Hermanas municipality asserts its medium size town status to affirm its independance from Seville.

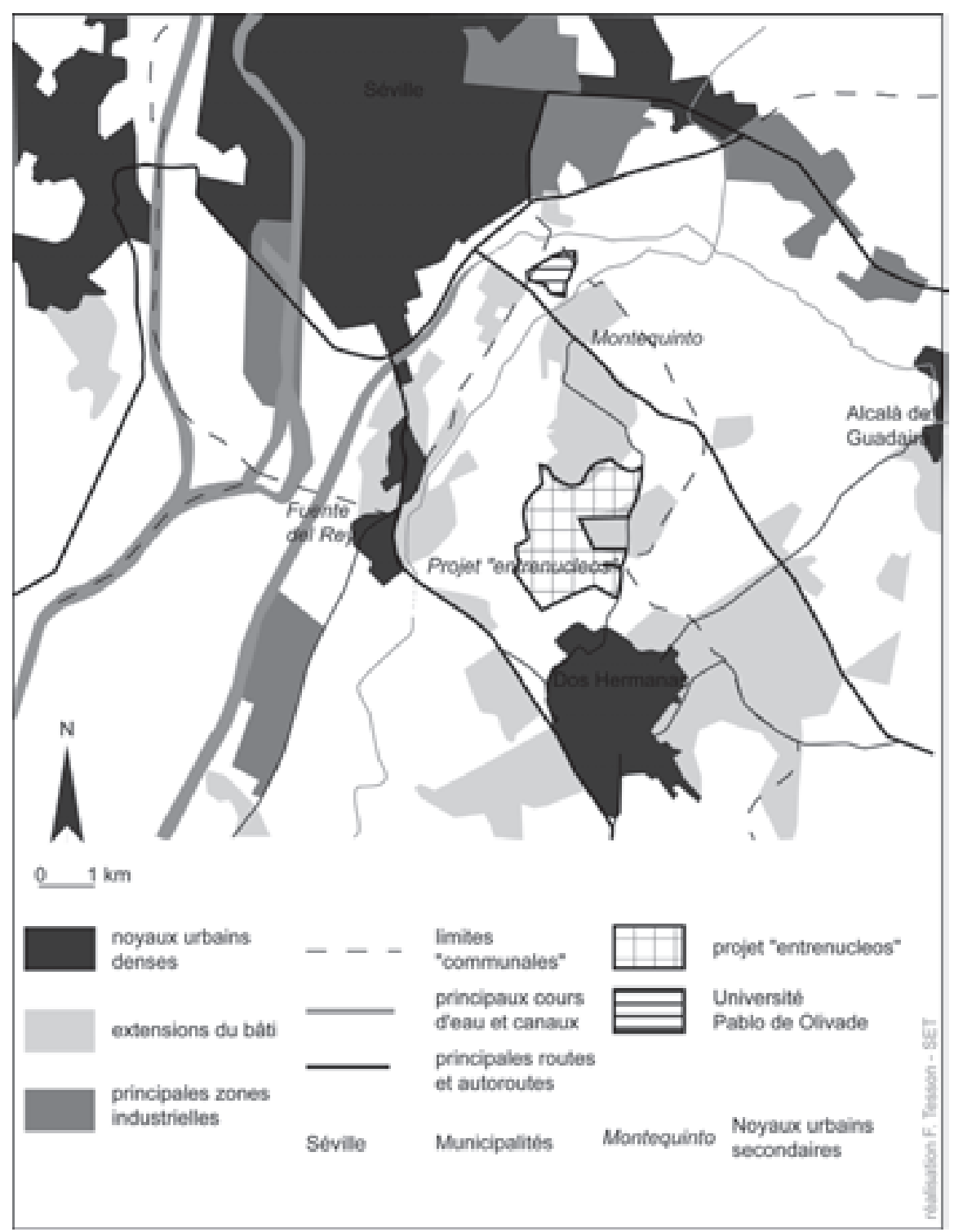

de donner une lisibilité aux incubateurs développés sur le territoire. Le projet principal de la ville dans le cadre du programme ATI était l'organisation du deuxième congrès " innovation et entreprise », qui s'est déroulé en septembre 2005 avec comme slogan "l'innovation ne peut pas attendre ».

Dans le cadre de l'enquête destinée à comprendre comment chaque partenaire voyait son implication dans le programme, les édiles nazarenos ont fourni des réponses particulièrement claires quant à l'objectif qu'ils poursuivaient. Pour eux l'innovation est technologique, l'attractivité se mesure à la capacité à attirer des entreprises et de la population, et l'échelle de la ville moyenne est celle de la municipalité.
Le contexte sévillan, très dynamique lui donne les atouts pour un développement économique permanent. Le problème de Dos Hermanas est un problème de foncier pour développer ses activités tout en préservant un certain cadre de vie et loger une population qui cherche à sortir du maelstrom urbain. Ainsi, de grosses opérations immobilières ont été lancées dans l'interstice qui sépare la ville de la capitale andalouse. Le projet «entrenucleos » doit réunir les deux villes dans une continuité urbaine qui, si elle correspond au sens de l'histoire et de la géographie, ne plaide pas pour une meilleure identification de Dos Hermanas dans l'espace métropolitain. 
Pourtant c'est sur ce point que la municipalité insiste lorsqu'elle aborde ce qui nuit à l'attractivité de Dos Hermanas. En effet, outre la mise en avant de la prédominance des PME qui constituent $90 \%$ des entreprises locales, c'est l'absence d'identité qui est stigmatisée. La proximité avec Séville joue à la fois comme une aubaine mais se révèle aussi une faiblesse, du fait du défaut de lisibilité qu'elle induit et de la domination qu'elle impose. La revendication concernant l'adresse postale de l'université Pablo de Olivade, située à $90 \%$ sur le territoire de la commune mais dont la domiciliation reste dans la capitale, témoigne de cette frustration permanente.

Ainsi, l'exemple de Dos Hermanas traduit bien le rôle que peut jouer l'affichage "ville moyenne ». La quête de reconnaissance et d'identification sur la scène européenne, conduit les acteurs de cette ville à s'affirmer «ville moyenne » pour exister dans le contexte métropolitain sévillan. Le jeu est complexe puisqu'à la fois ils construisent leur appartenance à l'aire métropolitaine en fabricant la continuité urbaine avec la ville centre, pour devenir incontournable à cette échelle, mais ils s'isolent au niveau européen pour apparaître en tant que ville moyenne auprès des partenaires et des investisseurs potentiels.

\section{Torrelavega comme satellite de Santander au sein de la conurbation cantabrique}

L'exemple de Torrelavega est un peu différent tout en révélant des caractéristiques relativement proches. En effet, La ville de Torrelavega compte environ 56000 habitants. Elle s'affirme comme la seconde ville de Cantabrie derrière la capitale Santander qui en compte près de 185000 . Mais distante de $25 \mathrm{~km}$ et reliée par autoroute, Torrelavega n'est en fait qu'un satellite de la capitale. L'INE associe même les deux municipalités pour former une conurbation. Celle-ci comptait 395000 habitants en 2009. De plus, même associées, les deux villes ont du mal à s'affirmer comme une métropole tant la proximité de l'aire métropolitaine quasi millionnaire de Bilbao, située à un peu plus d'une heure, interdit une réelle émancipation.

Cette description même du contexte urbain vient corroborer la thèse de l'artificialité de l'affirmation de Torrelavega en tant que ville moyenne pourtant développée par les édiles de la ville dans le cadre du programme INTERREG dont il est question ici. Là encore, comme pour Dos Hermanas, la quête d'identité fait partie des principaux éléments relevés comme défaillants, même si Torrelavega jouit d'une plus grande reconnaissance que la ville andalouse du fait d'un passé plus riche. Pour le reste, l'engagement de Torrelavega dans le programme reposait sur une affirmation du caractère innovant de son industrie et dans la promotion d'une pépinière d'entreprise particulièrement réussie.

On peut souligner également, pour l'anecdote, que la candidature de Torrelavega doit beaucoup au fait que les bureaux administratifs de l'ensemble des programmes INTERREG SUDOE était situés à Santander, et que l'accès à l'information leur a été facilité.

\section{Des indicateurs en creux : identité et domination}

Que nous disent ces affiliations artificielles sur la catégorie qui nous préoccupe ici? D'une part elles mettent en évidence la dimension identitaire des villes moyennes dont elles se sentent dépourvues. Ainsi, la connaissance et la reconnaissance de la ville moyenne en seraient des caractéristiques fondamentales. Elles renforcent également l'idée que la ville moyenne est certes dominée mais qu'elle domine également. Or cette seconde dimension, dans le cas des banlieues ou des satellites, n'est pas évidente. Elles permettent aussi de montrer, même si cela semble acquis, le caractère inopérant des seuils démographiques. Pour finir, on peut dire qu'elles donnent du crédit à une approche morphologique telle que la suggèrent les équipes de chercheurs engagées dans le programme ORATE/ ESPON 1.4.1. L'idée de travailler sur la morphologie, à l'aide d'outils tels Corine Land Cover, peut être intéressante en complément des analyses démographiques et fonctionnelles.

\section{Conclusion générale}

Nous avons pu mettre en évidence à travers ce regard porté sur un programme INTERREG consacré explicitement à la ville moyenne que, même si les attendus de l'appel à collaboration n'étaient pas neutres, il existait des stratégies bien distinctes, por- 
tées par des acteurs locaux aux profils différents, susceptibles de nous éclairer sur ce qu'est une ville moyenne.

Ainsi, il est apparu que la position dans la hiérarchie urbaine est un levier de détermination prépondérant lorsque les acteurs amenés à intervenir sur ou pour la ville moyenne appartiennent à des structures englobantes (régionales), et ce, même s'il s'agit d'institutions issues du monde économique (chambres de commerces). Ce regard porté sur la ville moyenne, la positionne relativement à sa distance topographique au centre. Ainsi, le critère démographique, prégnant dans la littérature scientifique sur le sujet, est donc à considérer relativement à la taille du centre et non de manière absolue. De fait, pour ces institutions essentiellement régionales, la ville moyenne serait celle qui se situe à distance de la ville centre et dans la catégorie démographique immédiatement inférieure. Il s'agirait donc de la ville dominée par le centre, mais qui domine un hinterland qui échappe, pour nombre de fonctions spécifiques, à la capitale régionale. L'attention toute particulière de ces institutions pour les villes de ce type témoigne d'une prise de conscience de la nécessité de multiplier les opérations "d'aménagement du territoire » envers une catégorie de ville que l'on perçoit importante, mais que l'on sent également fragile dans son attractivité. Si l'identité de ces villes est une force, elle ne suffit pas à les intégrer pleinement dans le marché mondialisé.

L'attractivité et l'identité sont, par ailleurs, deux points clés révélés par la seconde catégorie d'acteurs participant à ce programme. En effet, lorsqu'on observe les motivations des élus locaux qui ont engagé nommément leur propre ville dans l'aventure, on se rend compte que l'identité apportée par le statut de ville moyenne est prépondérante. Cette révélation apparaît en creux puisque les villes en question, Dos Hermanas et Torrelavega, ne font pas partie, comme nous l'avons vu, de ce que l'on peut considérer comme des villes moyennes, puisqu'elles sont banlieue pour la première, satellite pour la seconde, d'une capitale régionale. Leurs édiles, sans l'exprimer ainsi, en ont conscience puisque qu'ils considèrent que l'intégration à un programme INTERREG consacré aux villes moyennes peut leur apporter ce supplément d'âme qui, de leur propre aveu, leur fait défaut. Le décalage de ce regard sur la ville moyenne, posé par des élus qui sont a priori exclus de la catégorie tout en cherchant à l'intégrer, peut apparaître comme un gage de l'importance de cette identité qu'ils considèrent comme intrinsèque au statut de ville moyenne.

Ces deux composantes d'une définition de la ville moyenne, qui tente de s'appuyer sur ce que les acteurs en disent et en pensent, permettent de resserrer le débat et de le faire sortir de la seule dispute scientifique, parfois vaine. C'était le parti pris de notre propos qui, de ce fait, occulte en partie la richesse de ce que les chercheurs peuvent apporter au débat. Il est donc fondamental de considérer cette analyse comme un autre regard, en ayant conscience que celui-ci n'est pas totalement nouveau comme en témoignent les travaux menés par les acteurs régionaux, parmi lesquels on peut citer schéma de développement de l'espace atlantique proposé par la conférence des régions périphériques maritimes d'Europe (CRPM, 2005). Par ailleurs, du côté d'une recherche plus impliquée, le numéro consacré aux villes moyennes de l'Arc Atlantique par la revue Norois en 1996 (Norois, 1996) constitue également une référence. On trouve en effet dans cette livraison de la revue plusieurs articles rédigés par des acteurs parfois élus locaux et notamment deux pages introductives du maire de Cholet dont la réflexion sur la ville moyenne témoigne d'une grande sensibilité (Ligot, 1996) et préfigure le type de regard que nous avons souhaité porter ici.

\section{Bibliographie}

Alter N., 2005 [2000], L'innovation ordinaire, Paris, PUF, $284 \mathrm{p}$.

Christaller W., 2005 [1933], Les lieux centraux en Allemagne du Sud. Une recherche économico-géographique sur la régularité de la diffusion et du développement de l'habitat urbain (1933), Cybergeo, n³ 300, p. 1-17.

CRPM, 2005, Schéma de développement de l'espace Atlantique, Porto, CRPM, $51 \mathrm{p}$.

Datar, 1991, En Europe, des villes en réseaux, Paris, La documentation française, $109 \mathrm{p}$.

Diact, 2007, Les villes moyennes françaises, Paris, La documentation française, $64 \mathrm{p}$.

FAure, A. (2009). Les élus locaux changent-ils la politique?, dans Bidégaray C., Cadiou A. et Pina C. (dir.), L'élu local anjourd'hui, Grenoble, Presses Universitaires de Grenoble, p. 29-39.

Guichard O. (dir.), 1986, Propositions pour l'aménagement du territoire, Paris, La Documentation Française, 61 p 
Ligot, M., 1996, La ville moyenne et son pays, Norois, V. 43, $\mathrm{n}^{\circ} 171$, p. 479-480.

Norois, 1996, Les villes moyennes dans l'Arc Atlantique, $\mathrm{n}^{\circ} 171$.

Orate/Espon i.4.I, 2006, The Role of Small and MediumSized Towns (SMESTO) - 1.4.1, EPSON/ORATE, Vienne (Autriche), $435 \mathrm{p}$.

Santamaria F., 2000, La notion de «ville moyenne » en France, en Espagne et au Royaume-Uni, Annales de géographie, $n^{\circ}$ 613, p. 227-239.

Tesson F., 1996, Les réseaux de villes en France, recherche sur le rapport de l'élu local à l'espace, Thèse nouveau régime en géographie et aménagement. Piolle X. (dir.), université de Pau et des Pays de l'Adour. 399 p.
Tesson F., 2000, « Réseau de villes », in La ville et l'urbain, l'état des savoirs, Body-Gendrot S., Lussault M. et Paquot Th., Paris, La Découverte, p. 255-264.

Tesson F., 2010, Les « réseaux de villes » : l'intercommunalité du $\mathrm{XxI}^{\mathrm{e}}$ siècle, retour sur une belle idée... in Réseaux en question : utopies, pratiques et prospective. BLETON-RUGET A., Commerçon N., Vanier M., Mâcon, Insitut du Val de Saône-Mâconnais, p. 333-340.

Tesson F, 201 1, Villes moyennes, des réseaux toujours à l'ombre des métropoles? », Urbanisme, n 378, p. 47-49. 